\title{
QoS Mapping between User's Preference and Bandwidth Control for Video Transport
}

\author{
Kentarou Fukuda, Naoki Wakamiya, Masayuki Murata \\ and Hideo Miyahara \\ Department of Informatics and Mathematical Science \\ Graduate School of Engineering Science, Osaka University \\ Toyonaka, Osaka 560, Japan \\ Tel:+81-6-850-6588 Fax:+81-6-850-6589 \\ $\{k$-fukuda,wakamiya,murata,miyahara\}@ics.es.osaka-u.ac.jp
}

\begin{abstract}
In this paper, we present a method of QoS mapping between user's preference on video quality and a required bandwidth to transport the video across the network. We first investigate the mapping method from QoS parameters to the required bandwidth on the network. For this purpose, we assume that the underlying network supports some bandwidth allocation mechanism, such as DBR service class in ATM, RSVP, IPv6 and so on. Then, for given QoS parameters in terms of spatial, SNR, and timely resolutions, the required bandwidth to support the MPEG-2 video transmission is determined by analyzing the traced MPEG-2 streams. We next consider the mapping method between QoS parameters and the user's perceived video quality, which is quantified by MOS (Mean Opinion Score) evaluation. Based on the above results, we discuss a systematic method to estimate the required bandwidth to guarantee user's preference on video quality.
\end{abstract}

\section{Keywords}

QoS architecture, MPEG-2, bandwidth allocation, perceived quality

\section{INTRODUCTION}

A distributed multimedia system requires the QoS (Quality of Service) guarantee to achieve its effective presentation (Campbell et al. 1996). QoS guarantee is performed in each of entities within the multimedia communication system. As an example, in the Video on Demand (VoD) application, the video server is responsible for providing a multi-client real-time access to stored video libraries, continuous data emission, and an interaction mechanism with clients. The client provides users with the continuous and high quality video presentation and a means of QoS control to reflect user's preference on the perceived video quality. Further, the underlying transport network requires some mechanism for the distributed multimedia application to guarantee QoS requested by the users.

The distributed multimedia system should take account of user's preference. Some users may prefer slower, but detailed video appearance while other users may choose more coarse but faster video presentation. Those preferences on the video quality have to be mapped onto QoS parameters that each entity can understand (Figure 1), and all of them cooperate with each other to provide the required QOS on perceived video quality.

Video data coded by any coding algorithm (e.g. MPEG (ISO/IEC DIS 


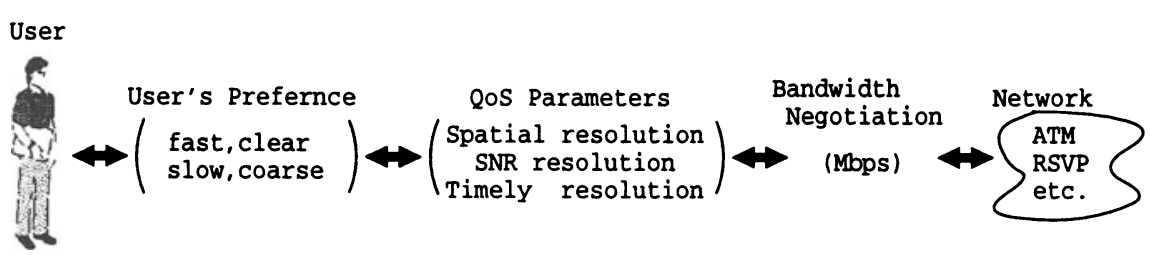

Figure 1 From users' preference to bandwidth

11172 1991)) are transferred over the transport network. The network is required to provide some bandwidth control mechanism to guarantee the required QoS for each connection. One example is the ATM (Asynchronous Transfer Mode) network, which is now a well-known transport network for an effective multimedia data transfer (The ATM Forum 1995). In ATM, service classes have been standardized to support various QoS guarantees in two standardization bodies, ITU-T and the ATM Forum (ITU-T 1992 revised in 1995, Garrett 1996). Among them, either SBR (Statistical Bit Rate) or DBR (Deterministic Bit Rate) is suitable for video data transfer with QoS guarantee since those two service classes have a capability to guarantee the transfer delay, delay jitter and cell loss ratio. Between two, the SBR service class seems to be more preferable because an effective bandwidth usage can be expected by means of statistical multiplexing, and QoS guarantees are provided. However, the SBR service class can only perform QoS guarantees statistically, and requires complicated UPC/CAC mechanisms (Krunz et al. 1995, Newman 1994). On the other hand, in the DBR service class, bandwidth allocation is performed based on PCR (Peak Cell Rate) and the deterministic QoS guarantee is provided as far as the cell emission rate is kept under the allocated bandwidth (Newman 1994). Other transport mechanisms such as RSVP (Braden et al. 1996) and IPv6 (Deering et al. 1995) also provide the bandwidth allocation mechanism to provide the deterministic QoS guarantees.

In those bandwidth allocation based networks, connection setup is performed by allocating the enough bandwidth to that connection. It means that the required bandwidth must be known or estimated a priori at the connection setup time. However, the bandwidth prediction for coded video traffic is known to be very hard since the traffic characteristics must depend on the coding algorithm and contents of the video sequence. It is especially true when the coding algorithm allows to adaptively encode the video according to the user's preference. If the coded video is stored in the server's storage, a completely accurate amount of the required bandwidth would be decided. However, under the heterogeneous environment, various kinds of QoS requirements would be required by clients and it becomes ineffective to prepare many video streams of various resolutions. Thus, the video server must adapt the coding method according to the user's preference. As a result, the characteristics of generated video traffic also vary.

Some researches have already been devoted to traffic prediction issues (see, e.g., (Singh et al. 1995, Heyman et al. 1996, Wu et al. 1995) and references therein), but their results are not applicable to the case discussed in the above. Especially when user's preference are taken into account, the prediction algorithms presented in those papers are not useful any longer, because they consider the behavior of video traffic coded with some specific parameter sets. For example, in (Yeadon et al. 1996), they investigated the effectiveness of 
several filtering control methods on the throughput of MPEG coded video streams. However, their approach does not provide the way to estimate the required bandwidth when users are allowed to set arbitrary values for QoS parameters.

From the network side, the network resource is limited. Thus, even when the traffic prediction can be performed accurately, the requested bandwidth may not be admitted by the network due to the lack of network resources. However, the reduced bandwidth may be accepted by the network if the quality degradation is allowed by the user. A problem is that we do not have any device to decide QoS parameters for a given bandwidth. Further, when the user dynamically changes QoS parameters during the connection, the allocated bandwidth should be re-computed and re-negotiated through the signaling protocol such as Q.2963 (ITU-T Draft Standard Q.2963 1995) or ABT (ATM Block Transfer) (Guillemin et al. 1996). When the enough bandwidth is not available and re-negotiation fails, it is possible that the user reduces the bandwidth, and then try the bandwidth reservation again using those protocols. A remaining problem is how to decide the reduced bandwidth while keeping little degradation in perceived video quality.

From above observations, one of most important issues in the distributed multimedia architecture is to investigate the relationship between QoS parameters and the required bandwidth (Figure 1), which is one of main subjects of this paper. As QoS parameters, we will consider spatial resolution (the number of pixels), SNR resolution (the quantization degree) and timely resolution (the number of frames per second) of MPEG-2. Then, we examine the effect of the scalable control mechanism (i.e., QoS parameter setting) of MPEG-2 coded video stream on the perceived video quality through MOS (Mean Opinion Value) evaluation. The obtained relationship is useful to decide the appropriate bandwidth at the call setup time and at the bandwidth re-negotiation time. Or, when the bandwidth is limited, the user can set the QoS parameters such that the perceived video quality is kept as high as possible. Of course, such a relationship is applicable to existing multimedia systems such as the one in (Chang et al. 1996).

This paper is organized as follows. We first briefly summarize the QoS parameters on the perceived video quality in Section 2. In Section 3, we investigate the quantitative relationship between QoS parameters and the required bandwidth. Further we investigate the influence of QoS parameter set on the perceived video quality in terms of MOS values in Section 4 . By combining those results, mapping from user's requested video quality to the required bandwidth can be established. It can be utilized when the user establishes the connection of video transfer as described in the previous section. Or, inverse mapping from the available bandwidth to user's perceived quality can also be built, which is useful for the flexible bandwidth re-negotiation mechanism between the user and the network. We will discuss this aspect in Section 5 .

\section{QOS PARAMETERS FOR MOTION VIDEO}

In this paper, we assume that user's preference can be related to QoS parameters in terms of spatial resolution scalability, SNR (Signal to Noise Ratio) resolution scalability, and timely resolution scalability of MPEG-2 videos, which will be briefly summarized in this section. 
Table 1 Video characteristics (640x480 pixels, $30 \mathrm{fps}, Q=10)$

\begin{tabular}{lcccc}
\hline sequence & Scenery & Starwars & Live & Comedy \\
\hline $\max (\mathrm{Mbps})$ & 14.373 & 10.715 & 9.085 & 11.192 \\
\hline mean (Mbps) & 4.063 & 4.919 & 2.428 & 4.889 \\
\hline burstiness & 3.538 & 2.178 & 3.742 & 2.289 \\
\hline
\end{tabular}

The spatial resolution of perceived video is described by the number of pixels in each frame. As the preferred spatial resolution, users may select $640 \times 480$ pixels, $320 \times 240$ pixels or $160 \times 120$ pixels. When the user receives the video streams of $640 \times 480$ pixels large, the user can enjoy the detailed and high quality video contents. When the received video is $320 \times 240$ pixels large, on the other hand, the user would suffer from the coarse and rough quality of video when it is enlarged on the TV monitor, or the smaller and degraded quality of video on the computer monitor. However, the required network bandwidth to transfer $320 \times 240$ video is certainly smaller than $640 \times 480$ video. In this paper, we will assume that the TV monitor is used, and henceforth, the frame size of $320 \times 240$ video is enlarged by four times when its perceived quality is compared with that of $640 \times 480$ video in Section 4 .

The SNR resolution scalability is realized by adjusting the degree of quantization during the video coding process. The quantizer scale can be adjusted without MPEG-2 codec with full capability since only de-quantization and quantization are required in this case. The quantization in the MPEG-2 coding algorithm is performed by applying specific quantizer scale against each block of $16 \times 16$ pixels large. When a larger quantizer scale is applied, the quality of decoded block becomes poorer, which leads to degraded SNR values. However, the coded block size can become smaller, which has a positive effect from a viewpoint of effective resource usage within the network.

The timely resolution of received video is related to the number of frames per second (fps). The frame rate of coded MPEG-2 video stream can be regulated by means of a frame dropping technique. MPEG-2 video stream consists of three types of frames, I (Intra coded), P (Predictive coded) and B (Bidirectionally coded) frames. One of the video sequence we use in this paper has a cyclic sequence of IBBPBB, which is called GoP (Group of Pictures). The frame rate can be reduced by dropping some frames. Since the least influential frames are B frames, we can drop B frames first. The resulting GoP structure then becomes I BP B and the frame rate is reduced by two thirds, i.e., from 30 fps to 20 fps. By displaying the preceded frame repeatedly, the empty frame time should be filled. As we will show in the below, it can be expected that the video quality is not degraded by dropping B frames. However, the required bandwidth cannot be reduced by doing so since the size of $\mathrm{B}$ frames are much smaller than other I or P frames. We will discuss this issue in more detail in Section 3.

In Sections 3 and 4, we will discuss QoS parameters of MPEG-2 video described in the above in relation to the required bandwidth and the user's perceived video quality, respectively. Before proceeding to those sections, we summarize the statistics of MPEG-2 coded video streams used in our investigation. We employ four different video streams which are coded from laser 


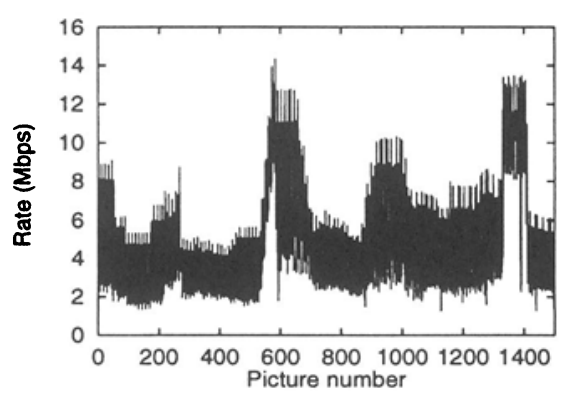

Figure 2 Time dependent behavior Figure 3 Peak rate of coded video of peak rate (Scenery)

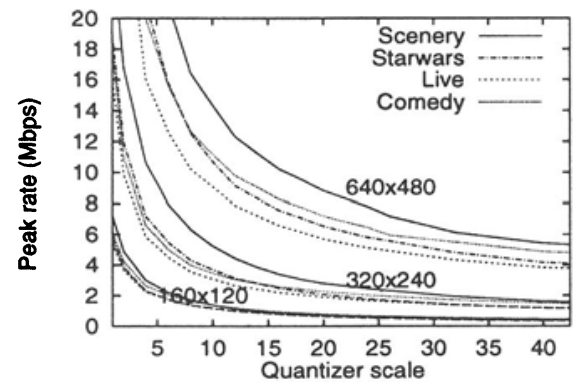

stream (30 fps)

disks. Those are Scenery, Starwars, Live and Comedy. Their characteristics are summarized in Table 1 and the rate transition of Scenery is depicted in Figure 2. In the table, we show the traffic rate in terms of Mbps and burstiness in terms of "peak to average ratio" for the case where the spatial resolution is set to be $640 \times 480$ pixels, the frame rate is 30 frames per second and the quantizer scale is 10 . For other QoS parameters, we will use $640 \times 480$ pixels, $320 \times 240$ pixels and 160x120 pixels for the video size. The maximum frame rate is 30 frames per second, and GoP structure is IBBPBB or IPPPPP. The quantizer scale will be chosen from a range of 1 (highest SNR) to 112 (lowest SNR). As shown in the tables and figures, the video characteristics are widely varied. Our main objective is then to find some common properties in those videos so that the result can be applied to handle MPEG-2 videos in multimedia systems with QoS guarantees.

\section{QOS PARAMETERS AND REQUIRED BANDWIDTH}

There are two alternatives in allocating the bandwidth to VBR traffic such as MPEG-2 coded video data. One is to allocate the bandwidth equal to the actual peak rate of the video stream. By this bandwidth allocation policy, all the data can be delivered to the destination without buffering delay and data loss. It is thus preferable for interactive applications where end-to-end transfer delay should be kept as small as possible. In this case, we employ the GoP structure of IPPPPP since coding of B frames requires the buffering of preceding and following frames for further effective data compression. When the application can tolerate large buffering delay at the source and/or within the network, on the other hand, the required bandwidth can be decreased by rate smoothing, which will be discussed later in this section.

In Figure 3, the peak rate of the coded video streams are presented dependent on the quantizer scale for different spatial resolutions (pixels). From the figure, we can observe that the absolute values of peak rate are different, but the same tendency is obtained independent on video content, spatial resolution and frame rate; as the quantizer scale becomes larger, the peak rate decreases. Further, the larger quantizer scale does not contribute to the decrease of the peak rate when it is beyond 35 . To see the above-mentioned relationship more clearly, the peak rate is normalized by the peak rate in the case where the quantizer scale is 10 . The result is shown in Figure 4. In obtaining this figure, the spatial resolution and the frame rate are set to be 640x480 pixels and $30 \mathrm{fps}$, respectively. From Figure 4, we can estimate the 


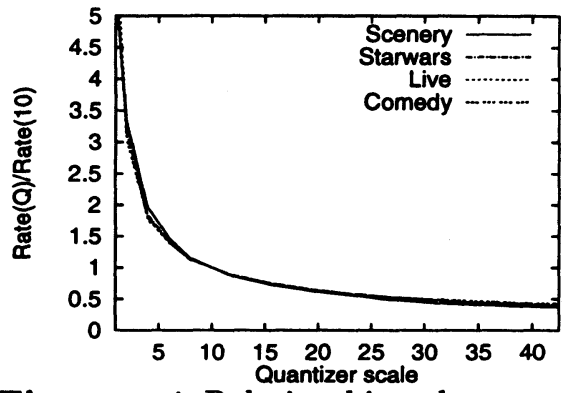

Figure 4 Relationship between quantizer scale and normalized peak rate $(640 \times 480$ pixels, $30 \mathrm{fps})$

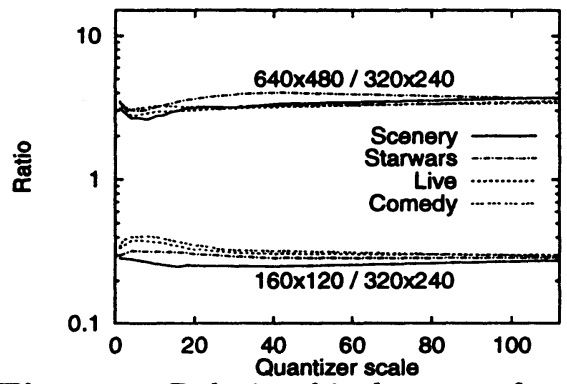

Figure 6 Relationship between frame size and peak rate ratio (30 fps)

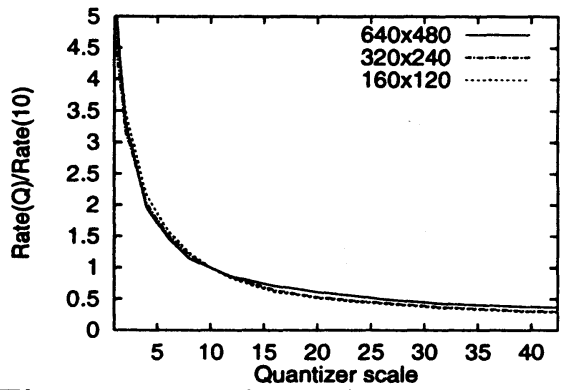

Figure 5 Relationship between quantizer scale and normalized peak rate (Scenery, $30 \mathrm{fps}$ )

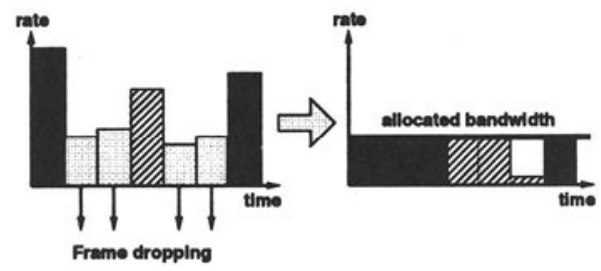

Figure 7 Frame dropping

peak rate of the video sequence for a given quantizer scale if we can know the peak rate of some quantizer scale. For example, if the peak rate for the quantizer scale of 10 is $10 \mathrm{Mbps}$, the peak rate for the quantizer scale of 20 must be about $7 \mathrm{Mbps}$. This relationship also holds even when the spatial resolution is changed as shown in Figure 5, where the effect of the spatial resolution on the relation between the quantizer scale and the peak rate is shown by using the video stream Scenery.

We next investigate the effect of the spatial resolution (the number of pixels in each frame) on the peak rate. Figure 6 plots (1) the ratio of the peak rate of $640 \times 480$ pixels video to that of $320 \times 240$ pixels video, and (2) the ratio of the peak rate of $160 \times 120$ pixels video to that of $320 \times 240$ pixels video. Those lines appear in the upper and lower region of the figure. The horizontal axis shows the quantizer scale. As shown in figure, the relationship is kept unchanged independent on the quantizer scale and the video content. The peak rate of $640 \times 480$ pixels video is about 3.1 times larger than that of $320 \times 240$ pixels video. This result is due to the MPEG-2 coding algorithm. When the number of pixels of each frame becomes four times larger, the number of blocks to compress also becomes four times larger. Since the header information must be attached with the coded frame data, however, the amount of coded data is only 3.1 times larger than that of the smaller video.

When the timely resolution is degraded by dropping one or more frames of GoP, the resulting peak rate decrease is inversely proportional to the number of dropped frames in the peak rate based bandwidth allocation. It is because by dropping $B$ frames, the empty time slots are generated, and henceforth, the transmission rate of $I$ and $P$ frames can be reduced as shown in Figure 7. Of 
course, as a result of frame dropping, the extra buffering delay is introduced and the perceived video quality in timely resolution must decrease.

We have found that there is a common tendency in the relationship between QoS parameters and the required bandwidth independently of the video content. First, the same relationship is held between the quantizer scale and the required bandwidth independently of the other QoS parameters. Second, there is another relationship between the spatial resolution and the required bandwidth. Third, the required bandwidth is inversely proportional to the number of dropped frames when the bandwidth is allocated based on the peak rate of video traffic. From these facts, we can estimate the required bandwidth from the QoS parameters as follows.

From Figures 4 and 5, we can express the relationship between the required bandwidth $B W_{Q}$ and the quantizer scale $Q$ as:

$B W_{Q} \cong\left(0.151+\frac{9.707}{Q}-\frac{4.314}{Q^{2}}\right) \times B W_{10}$

where $B W_{10}$ is the required bandwidth for the case where the quantizer scale being 10. The constants in Eq.(1) are chosen to fit the curve in Figure 4 (we usedfit function of Mathematica). From Figure 6, we observed that the required bandwidth for the video sequence becomes 3.1 times larger when the number of pixels is four times larger than the smaller video sequence. Furthermore, the required bandwidth is inversely proportional to the frame rate when the peak rate based bandwidth allocation is performed. Using Eq.(1), the required bandwidth $(B W(R, Q, F)[\mathrm{bps}])$ to guarantee the preferred video quality can be estimated as functions of spatial resolution ( $R$ [pixels]), the SNR resolution $(Q)$ and the timely resolution ( $F[\mathrm{fps}])$ as follows:

$B W(R, Q, F) \cong\left(\frac{1}{3.1}\right)^{\log _{4} \frac{R}{640 \times 480}}\left(0.151+\frac{9.707}{Q}-\frac{4.314}{Q^{2}}\right) \frac{F}{30} B W_{\text {base }}$

where $B W_{b a s e}$ is the required bandwidth for the case of $(R, Q, F)=(640 \times$ $480,10,30)$. For example, inScenery, since $B W_{b a s e}$ is $14.373 \mathrm{Mbps}$ for $(R, Q, F)$ $=(640 \times 480,10,30)$, we can depict Figure 9 from Eq. (2). As shown in the figure, we can accurately estimate the required bandwidth for any set of QoS parameters from Eq. (2). Although not shown in the figure, Eq.(2) is also applicable to the other MPEG-2 video streams, Starwars, Live and Comedy.

\section{QOS PARAMETERS AND PERCEIVED VIDEO QUALITY}

In the previous section, we have shown the relationship between QoS parameters and the required bandwidth. In this section, the relationship between QoS parameters and the user's preference on perceived video quality is investigated. By this way, we can know how the user's perceived video quality can be mapped into the bandwidth, and vice versa.

The perceived video quality is quantified through user's subjective evaluation by using MOS (Mean Opinion Score). Each testee gives a score from 1 (Poor) to 5 (Excellent) to the video sequence in experiments. Those scores are then gathered, and the MOS value is determined as

$M O S=\sum_{i=1}^{5} i \times \frac{\text { number of persons who give score } i}{\text { number of testees }}$ 


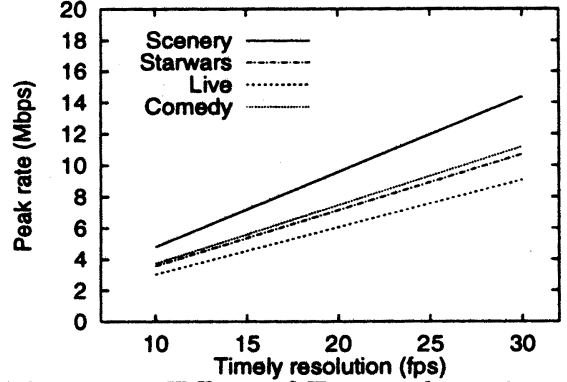

Figure 8 Effect of Frame dropping

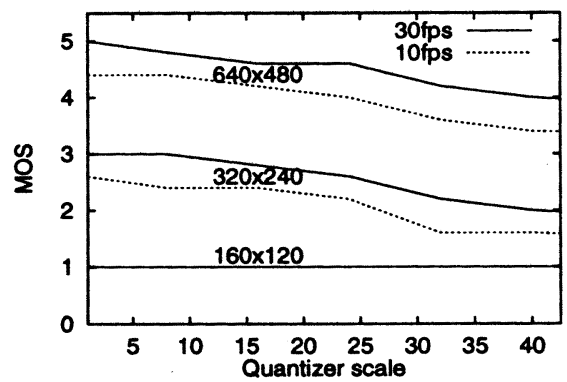

Figure 10 MOS evaluation (Scenery)

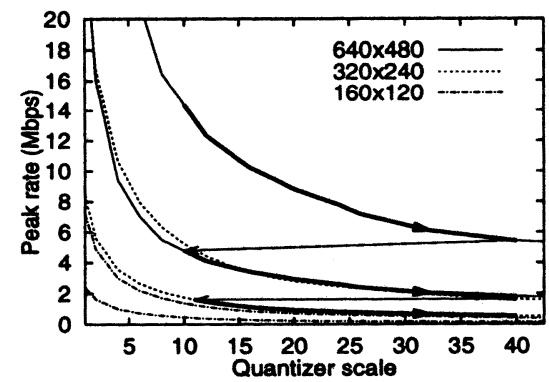

Figure 12 Effect of QoS control on peak rate

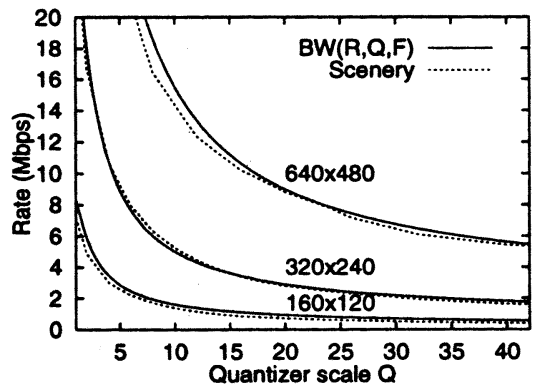

Figure 9 Comparison between estimated rate and actual rate (Scenery)

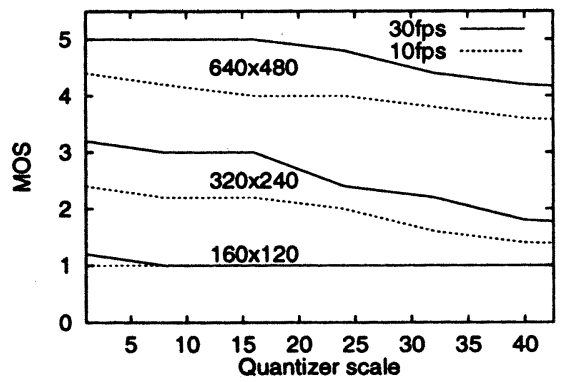

Figure 11 MOS evaluation (Comedy)

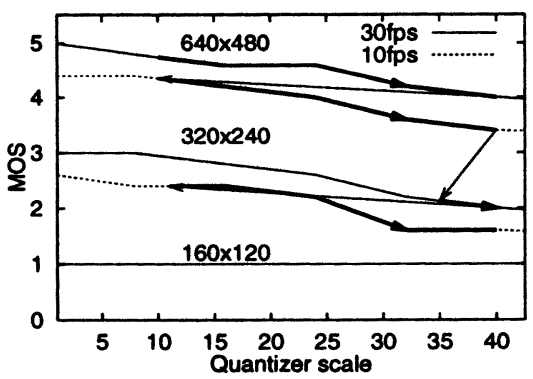

Figure 13 Effect of QoS control on MOS

Five testees participated in the experiments. In experiments, we assume that the received video is shown on the TV monitor and every frames are enlarged to $640 \times 480$ pixels large.

In Figures 10 and 11, we show the results of MOS evaluation on Scenery and Comedy for various sets of the spatial resolution $(640 \times 480,320 \times 240$ and $160 \times 120$ pixels), the SNR resolution (quantizer scale from 1 to 43) and the timely resolution (10 and $30 \mathrm{fps}$ ). As shown in the figures, reduction of the spatial resolution results in significant degradation of the perceived video quality. The $160 \times 120$ video sequences achieve the lowest subjective quality and quality variation is indistinguishable. When the timely resolution is reduced from 30 to $10 \mathrm{fps}$, the MOS value decreases since frame dropping decreases the smoothness of video presentation. The effect of reduction of timely resolution in Comedy (Figure 11) is a little bit larger than in Scenery (Figure 10). This 
result comes from the fact that Scenery consists of scenes with slow-moving while Comedy is made up of highly active scenes.

By taking into account the required bandwidth for given QoS parameters (Section 3), we obtain the following observation. Reducing the SNR resolution is a most effective way to decrease the required bandwidth while keeping the perceived video quality as high as possible. For example, the peak rate can be reduced to about a half when the quantizer scale is changed from 10 to 35 as shown in Figure 3. On the other hand, degradation in the MOS value can be kept to be no more than one in this case. However, an increase of the quantizer scale to more than 40 has no effect in saving the bandwidth. Further, reduction of the quantizer scale to less than 10 has no effect in increasing the perceived video quality. Henceforth, we should decrease the timely resolution next if the buffering delay induced by frame dropping is allowed. If further reduction of bandwidth is necessary, the spatial resolution should be reduced.

As an example of this QoS control mechanism, we consider the case of Scenery. We assume that the quantizer scale ranges from 10 to 40 , the timely resolution is either 10 or $30 \mathrm{fps}$ and the spatial resolution is $640 \times 480,320 \times 240$ or $160 \times 120$ pixels. Suppose that the user first requests the highest QoS, i.e., $(R, Q, F)$ is determined as $(640 \times 480,10,30)$. Then, the required bandwidth becomes 14.373 Mbps. When the required bandwidth is not available in the network, the SNR resolution is first decreased to reduce the bandwidth. At this time, other resolutions are kept to be unchanged. It results in the reduction of bandwidth from 14.373 Mbps to 5.414 Mbps as shown in Figure 12 by the topmost arrow. However, the perceived video quality is also decreased as shown in Figure 13 by the topmost arrow. When the quantizer scale becomes 40 , the timely resolution is degraded from 30 to $10 \mathrm{fps}$. When decreasing the timely resolution, we increase the SNR resolution again to maintain the perceived video quality as high as possible as shown in Figures 12 and 13. If further decrease of bandwidth is necessary, the SNR resolution is degraded again, and then the spatial resolution is degraded. With this QoS control mechanism, the required bandwidth can be decreased while keeping the perceived video quality as high as possible.

\section{BANDWIDTH ALLOCATION MECHANISMS WITH QOS GUARANTEES}

In what follows, we will explain how our results presented in Sections 3 and 4 can be applied to the distributed multimedia communication architecture for providing users' preference (QoS) guarantees. An abstract model of the communication architecture is illustrated in Figure 14. A similar architecture can be found in (Chang et al. 1996) and we believe that our results obtained in the current paper are also applicable to such an architecture. In the figure, the video data flow is depicted by the solid line. For the control messages, another connection is required as depicted by the dashed line in the figure

When the user establishes the multimedia connection, he/she first sets up the connection to the server with control messages. Together with the control message, he/she describes his/her preference on the perceived video quality by means of QoS control panel as shown at the top righthand corner in Figure 14. In the figure, user's preference is directly associated with a set of QoS parameters; the desirable spatial, $\mathrm{SNR}$ and timely resolution $(R, Q, F)$. How- 


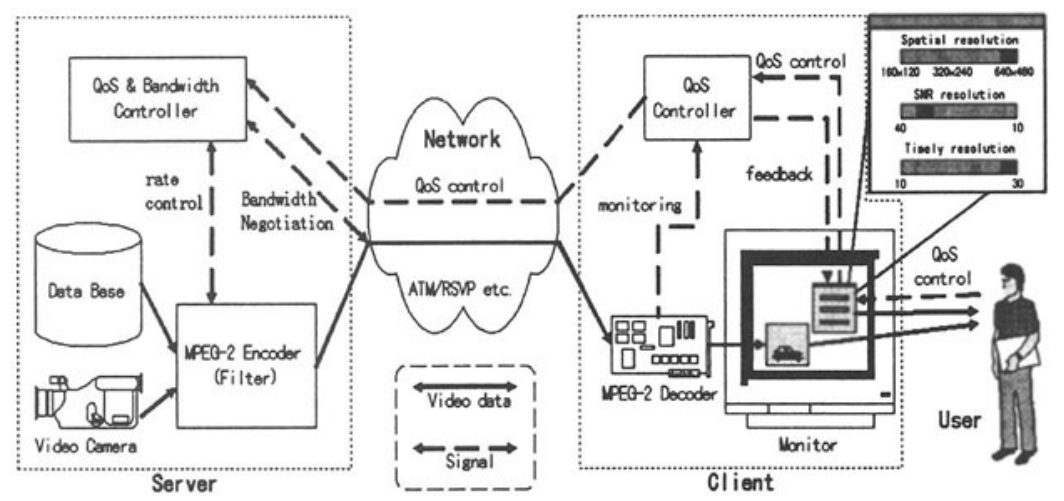

Figure 14 Distributed multimedia communication architecture

ever, more abstract representation is also applicable. For example, the user may want to select the degree of smoothness of the motion video. In that case, the results obtained in Section 4 can be used for more user-friendly interface.

In either way, QoS parameters decided by the user are sent to the server, and it decides whether the user's preference is acceptable or not. If the sender can provide the user with the video data of user's specified QoS parameters, then it determines the required amount of the bandwidth from the set of QoS parameters, which has been discussed in Section 3. Note that if the bandwidth reservation is performed in the receiver oriented fashion as in RSVP, a mapping from QoS parameters to the amount of bandwidth should be performed at the client. Eq. (2) is applicable only if we can estimate the peak rate of the video with a single set of QoS parameters. This is an easy task when the video is coded and stored at the server.

However, Video encoding may be performed in a real-time fashion. In that case, we do not have any information about the characteristics of the video sequence. Then, at the connection setup time, some reasonable value must be used as $B W_{b a s e}$ in Eq. (2). This value should be large enough to guarantee the required preference, but the larger amount of bandwidth results in the larger failure probability in bandwidth reservation. When $B W_{\text {base }}$ is inappropriately decided and the inadequate bandwidth is allocated, the bandwidth re-negotiation protocol (e.g. ABT) can be utilized. And $B W_{\text {base }}$ can be dynamically regulated to fit the actual coded video traffic as described later.

If the server cannot provide the user with the video of the required preference, or if the required bandwidth is not available within the network, then the QoS parameters must be decided again. To reduce the required amount of bandwidth, one or more QoS parameters should be degraded in the way that is described in Section 4. The user is informed with this QoS adaptation on the QoS control panel through the QoS query component.

After the bandwidth allocation is successfully performed, the compressed video stream is transferred from the server to the client over the allocated bandwidth. The received video stream is de-compressed by the MPEG-2 decoder and displayed on the monitor. The resolution of the displayed video is monitored by the QoS query component. By applying the information about the receiving video resolution and the amount of allocated bandwidth to Eq. 2, $B W_{\text {base }}$ is dynamically adapted to fit the actual characteristics of receiving video data. When the monitored resolution does not satisfy the user's prefer- 
ence because of the inappropriately allocated bandwidth, or the user dynamically changes the preference on the perceived video quality, the allocated bandwidth is re-negotiated by means of the appropriate signaling protocols such as Q.2963 or ABT. In the case of RSVP where dynamic QoS re-negotiation is allowed, the bandwidth re-negotiation is performed through the reservation messages (RESV) (Braden et al. 1996).

\section{CONCLUSION}

In this paper, we have presented the relations between QoS parameters and the required bandwidth presented in Section 3 and from QoS parameters and user's perceived quality in Section 4. From these results, we have obtained the QoS mapping method between user's preference on video quality and a required bandwidth to transport the video across the network. We use MPEG-2 as a video coding system in this paper. However, we think our QoS mapping method is also applicable to the other coding systems which employ DCT algorithm, such as H.261 or Motion JPEG. It can be applied to design the distributed multimedia communication architecture with perceived video quality supports. It is demonstrated by illustrating the example. Our result can also be useful for the heterogeneous environment in the case where the multiple client requests different QoS. It can be implemented by utilizing the QoS aggregation technique, but the description is omitted due to space limit.

\section{REFERENCES}

Braden, R., Zhang, L., Berson, S., Herzog, S. \& Jamin, S. (1996), 'Resource reservation protocol (RSVP) - version 1 functional specification', Internet Draft .

Campbell, A., Aurrecoechea, C. \& Hauw, L. (1996), 'A review of QoS architecture', ACM Multimedia Systems .

Chang, S., Eleftheriadis, A. \& Anastassiou, D. (1996), 'Development of Columbia's video on demand testbed', Image Communication Journal .

Deering, S. \& Hinden, R. (1995), 'Internet protocol, version 6 (IPv6) specification', Internet Draft/RFC1883 .

Garrett, M. W. (1996), 'A service architecture for ATM: From applications to scheduling', IEEE Network 10(3), 6-14.

Guillemin, F. \& Boyer, P. (1996), 'ATM block transfer capabilities: The special case of ABT/DT', Proceedings of IEEE GLOBECOM '96 pp. 762-766.

Heyman, D. P. \& Lakshman, T. V. (1996), 'What are the implications of longrange dependence for VBR-video traffic engineering?', IEEE/ACM Transactions on Networking 4(3), 301-317.

ISO/IEC DIS 11172 (1991), 'Coding of moving pictures and associated audio for digital storage media at up to about $1.5 \mathrm{Mbits} / \mathrm{s}$ ', ISO standard .

ITU-T (1992 revised in 1995), 'ITU-T recommendation I.371, traffic control and congestion control in B-ISDN', International Telecommunication Union .

ITU-T Draft Standard Q.2963 (1995), Preliminary Draft, International Telecommunication Union.

Krunz, M., Sass, R. \& Hughes, H. (1995), 'Statistical characteristics and 
multiplexing of MPEG streams', Proceedings of IEEE INFOCOM'95 pp. 455-462.

Newman, P. (1994), 'Traffic management for ATM local area networks', IEEE Communication Magazine 32, 44-51.

Singh, S. \& Chan, S. (1995), 'A multi-level approach to the transport of MPEG-coded video over ATM and some experiments', Proceedings of IEEE GLOBECOM'95 pp. 1920-1924.

The ATM Forum (1995), 'Traffic management specification version 4.0', ATM Forum 95-0013R9.

Wu, J. C., Chen, Y. \& Jiang, K. (1995), 'Modeling and performance study of MPEG video sources over ATM networks', Proceeding of IEEE ICC'95 pp. 1747-1750.

Yeadon, N., Garcia, F., Hutchison, D. \& Shepherd, D. (1996), 'Filters: Qos supoort mechanisms for multipeer communications', IEEE Journal on Selected Areas in Communications 14(7), 1245-1262.

\section{BIOGRAPHY}

Kentarou Fukuda: He is currently a master course student at the Department of Information and Computer Sciences, Osaka University, Osaka, Japan. His research work is in the area of video transfer in high-speed networks.

Naoki Wakamiya: received the M.E. and Ph.D. degrees in Information and Computer Sciences from Osaka University, Osaka, Japan in 1994 and 1996, respectively. From April 1996 to March 1997, he was an Assistant Professor in the Department of Information and Computer Sciences, Faculty of Engineering Science, Osaka University. On April 1997, he moved to the Education Center for Information Processing, Osaka University. His research interests include the performance evaluation of B-ISDN network. Dr. Naoki is a member of the IEEE.

Masayuki Murata: received the D.E. degree in Information and Computer Sciences from Osaka University, Japan, in 1988. In April 1984, he joined Tokyo Research Laboratory, IBM Japan, as a Researcher. From September 1987 to January 1989, he was an Assistant Professor with Computation Center, Osaka University. On February 1989, he moved to the Department of Information and Computer Sciences, Faculty of Engineering Science, Osaka University, and he has been an Associate Professor since December 1992. His research interests include computer communication networks, performance modeling and evaluation, and queueing systems. He is a member of the IEEE and ACM.

Hideo Miyahara: received the D.E. degree from Osaka University, Japan in 1973. From 1973 to 1980, he was an Assistant Professor in Kyoto University. From 1980 to 1986, he was an Associate Professor in the Faculty of Engineering Science, Osaka University. From 1986 to 1989, he was a Professor of the Computation Center, Osaka University. Since 1989, he has been a Professor in the Faculty of Engineering Science, Osaka University. From 1995, he is a director of Computation Center of Osaka University. His research interests include performance evaluation of computer communication networks and multimedia systems. He is an IEEE fellow. 